\title{
O conhecimento dos acadêmicos de medicina sobre a Oxigenação por Membrana Extracorpórea (ECMO) em pacientes com COVID-19
}

\author{
The knowledge of medical students about Extracorporeal Membrane Oxygenation (ECMO) in \\ patients with COVID-19 \\ El conocimiento de estudiantes de médico sobre la Oxigenación de Membrana Extracorporeal \\ (ECMO) en pacientes con COVID-19
}

Recebido: 28/10/2021 | Revisado: 07/11/2021 | Aceito: 16/11/2021 | Publicado: 25/11/2021

\author{
Delcio Uezato Junior \\ ORCID: https://orcid.org/0000-0002-7815-0901 \\ Universidade Municipal de São Caetano do Sul, Brasil \\ E-mail: delciouezatojunior@gmail.com \\ Amanda Costa Araujo \\ ORCID: https://orcid.org/0000-0003-2740-8252 \\ Universidade Municipal de São Caetano do Sul, Brasil \\ E-mail: amanda.araujo@online.uscs.edu.br
}

\begin{abstract}
Resumo
Objetivos: Avaliar o conhecimento dos médicos em formação sobre ECMO e ofertar um ciclo de palestras acerca das suas principais particularidades e aplicação na atual pandemia do COVID-19. Metodologia: Trata-se de um estudo prospectivo com aplicação de um questionário elaborado pelos autores antes e após a realização de um ciclo de palestras sobre ECMO e sua utilização nos pacientes com COVID-19. Foram comparadas as respostas dos acadêmicos antes e após a realização do ciclo de palestras ofertado pelos autores. Resultados: Calculando-se o ponto médio da soma de pontos obtidas no questionário pré e pós ciclo de palestras observa-se que sem o ciclo de palestras o ponto médio de acertos foi de 10,83, já após a realização do ciclo de palestras passou para 14,91. O teste de Wilcoxon comprovou uma diferença estatística entre os pontos obtidos no questionário pré e pós a realização do curso ( $\mathrm{p}=$ 0,039). Conclusão: Percebe-se a importância da inserção de temas extracurriculares na formação médica, principalmente a respeito de assuntos atuais, como é o caso de novas abordagens terapêuticas para pacientes com COVID-19.
\end{abstract}

Palavras-chave: ECMO; Oxigenação por membrana extracorpórea; COVID-19; Ensino; Palestra.

\begin{abstract}
Objectives: To assess the knowledge of medical students on ECMO and to offer a cycle of lectures about its main particularities and application in the current COVID-19 pandemic. Methods: This is an prospective research with application of a questionnaire prepared by the authors before and after a cycle of lectures on ECMO and its use in patients with COVID-19. The responses of academics were compared before and after the cycle of lectures offered by the authors. Results: Calculating the average point of the sum of points obtained in the questionnaire before and after the lecture cycle, it is observed that without the lecture cycle the average point of correct answers was 10.83, whereas after the lecture cycle it went to 14.91 . The Wilcoxon test proved a statistical difference between the points obtained in the questionnaire before and after the course $(\mathrm{p}=0.039)$. Conclusion: The importance of inserting extracurricular themes in medical education can be seen, especially regarding current issues, such as the case of new therapeutic approaches for patients with COVID.
\end{abstract}

Keywords: ECMO; Extracorporeal membrane oxygenation; COVID-19; Teaching; Lecture.

\section{Resumen}

Objetivos: Evaluar los conocimientos de los médicos en formación en ECMO y ofrecer un ciclo de conferencias sobre sus principales particularidades y aplicación en la actual pandemia de COVID-19. Metodología: Se trata de estudio prospectivo con la aplicación de un cuestionario elaborado por los autores antes y después de un ciclo de conferencias sobre ECMO y su uso en pacientes con COVID-19. Se compararon las respuestas de los académicos antes y después del ciclo de conferencias ofrecidas por los autores. Resultados: Calculando el punto medio de la suma de puntos obtenidos en el cuestionario antes y después del ciclo lectivo, se observa que sin el ciclo lectivo el promedio de aciertos fue de 10,83, pero después del ciclo lectivo pasó a 14,91. La prueba de Wilcoxon demostró una diferencia estadística entre los puntos obtenidos en el cuestionario antes y después del curso $(p=0,039)$. Conclusión: Se percibe la importancia de incluir temas extraescolares en la educación médica, especialmente en temas de actualidad, como el caso de nuevos abordajes terapéuticos para pacientes con COVID-19.

Palabras clave: ECMO; Oxigenación por membrana extracorpórea; COVID-19; Enseñando; Conferencia. 


\section{Introdução}

A oxigenação por membrana extracorpórea (ECMO) pode ofertar oxigênio parcial ou totalmente por meio de um suporte cardiopulmonar prolongado em que o sangue é drenado do seu leito nativo por meio de cânulas e devolvido à circulação após passar em um oxigenador por membrana e um trocador de calor (Tramm et al., 2015). É uma técnica que conduz a circulação do sangue através de cateteres e de bombas, onde o sangue sofre oxigenação artificial de forma direta (Amib, 2020).

Esse sistema pode ser rapidamente implantado e fornece suporte de oxigênio por meses. Nos pacientes com insuficiência respiratória grave, a ECMO é instalada na forma veno-venosa, ou seja, acessa e retorna o sangue pelo leito venoso, fornecendo troca gasosa não pulmonar e com isso protege o pulmão e oferece o tempo necessário para a sua recuperação (Forrest et al., 2011). Ela também pode ser instalada na forma veno-arterial fornecendo circulação sistêmica e prevenindo lesões isquêmicas secundárias a uma insuficiência cardíaca grave, por exemplo, e mais uma vez proporcionando a chance do órgão se recuperar ou ainda, servindo de ponte para um transplante cardíaco (Allen, Holena, McCunn, Kohl, Sarani, 2011). Ao oxigenar o sangue externamente a ECMO permite que o sangue oxigenado melhore a condição clínica do paciente (Ramanathan et al, 2020).

O primeiro relato bem-sucedido da utilização da ECMO foi descrito em 1972 pelo doutor Hill. Na ocasião o sistema foi implantado de forma veno-arterial em um paciente que desenvolveu insuficiência respiratória quatro dias após uma cirurgia de dissecção da aorta torácica (Hill et al., 1972). Após esse relato inúmeros outros foram descritos e o procedimento foi se expandindo em vários países. No entanto, embora em diversos países a ECMO seja uma realidade, no Brasil ainda existem inúmeras limitações para sua utilização, com apenas 21 centros registrados na Sociedade Internacional Extracorporeal Life Support Organization (ELSO), entidade responsável pelos protocolos de utilização, registro de uso e elaboração de pesquisa em nível mundial (Latam, 2020).

Nesse contexto, o restrito número de centros registrados no Brasil faz com que poucos médicos conheçam e possam oferecer esse recurso aos pacientes em estado grave, como o que vem ocorrendo frequentemente na atual pandemia do COVID-19. Com isso, esse estudo busca avaliar o conhecimento dos médicos em formação sobre essa tecnologia em ascensão, e ofertar um ciclo de palestras acerca das suas principais particularidades e aplicação na atual pandemia do COVID-19.

Apesar de uma proporção significativa dos pacientes diagnosticados com COVID-19 evoluírem sem nenhuma ou com poucas complicações respiratórias, aproximadamente 15 a 20\% dos doentes podem evoluir com formas mais graves da doença, necessitando de terapia intensiva e suporte ventilatório em decorrência da síndrome da angústia respiratória aguda (SARA) (Cunningham; Goh; Koh, 2020). Até 26,1\% dos pacientes abordados numa pesquisa necessitaram de cuidados intensivos, destes $61,1 \%$ evoluíram com SARA. A taxa de mortalidade nos casos críticos pode chegar a $61,5 \%$, e nenhum tratamento efetivo foi comprovado até então (Hong, Xiong, Feng, Shi., 2020; Zeng et al., 2020). Já em casos de hipoxemia refratária, a condução dos casos se torna ainda mais difícil, uma vez que todas as possibilidades com capacidade cientificamente comprovadas são escassas (Ramanathan et al, 2020; Patel, 2018).

Segundo a OMS a utilização da ECMO pode ser utilizada em pacientes com hipoxemia refratária além das medidas de cuidados usuais, desde que seja realizada em centros com experiência no manejo de ECMO (WHO, 2020). E, além disso, a ECMO é uma opção para pacientes com sinais de disfunção orgânica secundária ao menor aporte de oxigênio (Barros, Rivetti, Furnaletto, Teixeira, Welikow, 2020). Contudo, a ECMO pode apresentar efeitos colaterais importantes (Kon et al., 2021; Marodin, Goldin 2009), como alterações sanguíneas como plaquetopenia (Beyls, Huette, Abou-Arab, Berna, Mahjoub, 2020) e degradação de fibrinogênio (Huette et al., 2020) e bradicardia (Yang et al., 2020). Nessa perspectiva, terapias de suporte avançado com evidência científicas ainda não completamente estabelecida no COVID-19, como as técnicas de suporte cardiorrespiratório, devem ter seu risco-benefício avaliado (Ramanathan et al, 2020). 
Frente a constatação do diminuto número de centros registrados e capacitados para a utilização da ECMO, acredita-se que poucos acadêmicos de medicina tenham um conhecimento sólido sobre esse assunto. Desta forma, o objetivo desse estudo foi analisar o conhecimento dos acadêmicos de medicina sobre as principais particularidades da utilização da oxigenação por membrana extracorpórea (ECMO) e oferecer um ciclo de palestras sobre o uso da ECMO na atual pandemia do COVID-19.

\section{Metodologia}

\subsection{Tipo de estudo}

Estudo prospectivo com aplicação de questionário antes e após a realização de um ciclo de palestras sobre as particularidades e indicações da utilização da ECMO e sua aplicação em pacientes com COVID-19 (Estrela, 2018).

\subsection{Aspectos Éticos}

O presente estudo foi aprovado pelo comitê de ética com o número CAAE: 37267620.1.0000.5510, de acordo com as normas da Resolução 466/12 do Conselho Nacional de Saúde sobre pesquisa envolvendo seres humanos. Todos os participantes, após serem convenientemente informados sobre a proposta do estudo e procedimentos aos quais serão submetidos, assinaram o Termo de Consentimento Livre e Esclarecido - TCLE.

\subsection{Análise dos riscos e benefícios}

Riscos: A metodologia desse estudo consistiu na aplicação de um questionário e realização de um ciclo de palestras sobre o assunto estudado, tendo objetivo educativo. No entanto, todo estudo, mesmo que mínimo, oferece riscos aos participantes. Como por exemplo, invasão de privacidade, desconforto e/ou constrangimento para responder ao questionário, tomar o tempo dos participantes e/ou cansaço ao responderem ao questionário e ao assistirem as palestras. Sendo assim, para diminuir os possíveis riscos, foi garantido sigilo dos dados e caso o voluntário se sinta desconfortável e/ou constrangido em responder alguma questão pode desistir de participar do estudo a qualquer momento.

Benefícios: A realização e conclusão desse estudo proporcionou a ampliação do conhecimento médico científico de estudantes de medicina sobre esse tratamento em ascensão na atualidade.

\subsection{Local e população do estudo}

Este estudo foi realizado totalmente de forma online. O questionário, o conteúdo programático das palestras e o TCLE foram enviados por e-mail e as palestras foram realizadas via Google Meet. A população foi de alunos do primeiro ao sexto ano do curso de medicina da Universidade Municipal de São Caetano do Sul, campus São Paulo. O número amostral foi de 80 alunos. As palestras tiveram uma duração estimada de 10 minutos cada, sendo um total de 2 palestras.

\subsection{Delineamento do estudo}

Esse projeto seguiu as seguintes etapas:

a) Os alunos do primeiro ao sexto ano do curso de medicina foram convidados via e-mail a participar desse estudo e aqueles que concordaram enviaram o TCLE e o questionário pré-teste respondido para o email informado;

b) Foi ofertado um ciclo de 2 palestras online via Google Meet para os alunos que aceitaram participar do projeto e que enviaram os documentos listados acima. O conteúdo programático do ciclo de palestra consta no desse arquivo;

c) Após a finalização do conteúdo programático, foi reaplicado o questionário, a fim de verificar o conhecimento adquirido pelos alunos. 
d) Os alunos que não conseguiram participar do primeiro ciclo de palestras, tiveram uma nova, e última, data para assistirem as palestras. A Figura 1 ilustra o delineamento proposto nesse estudo:

Figura 1 - Delineamento do estudo.

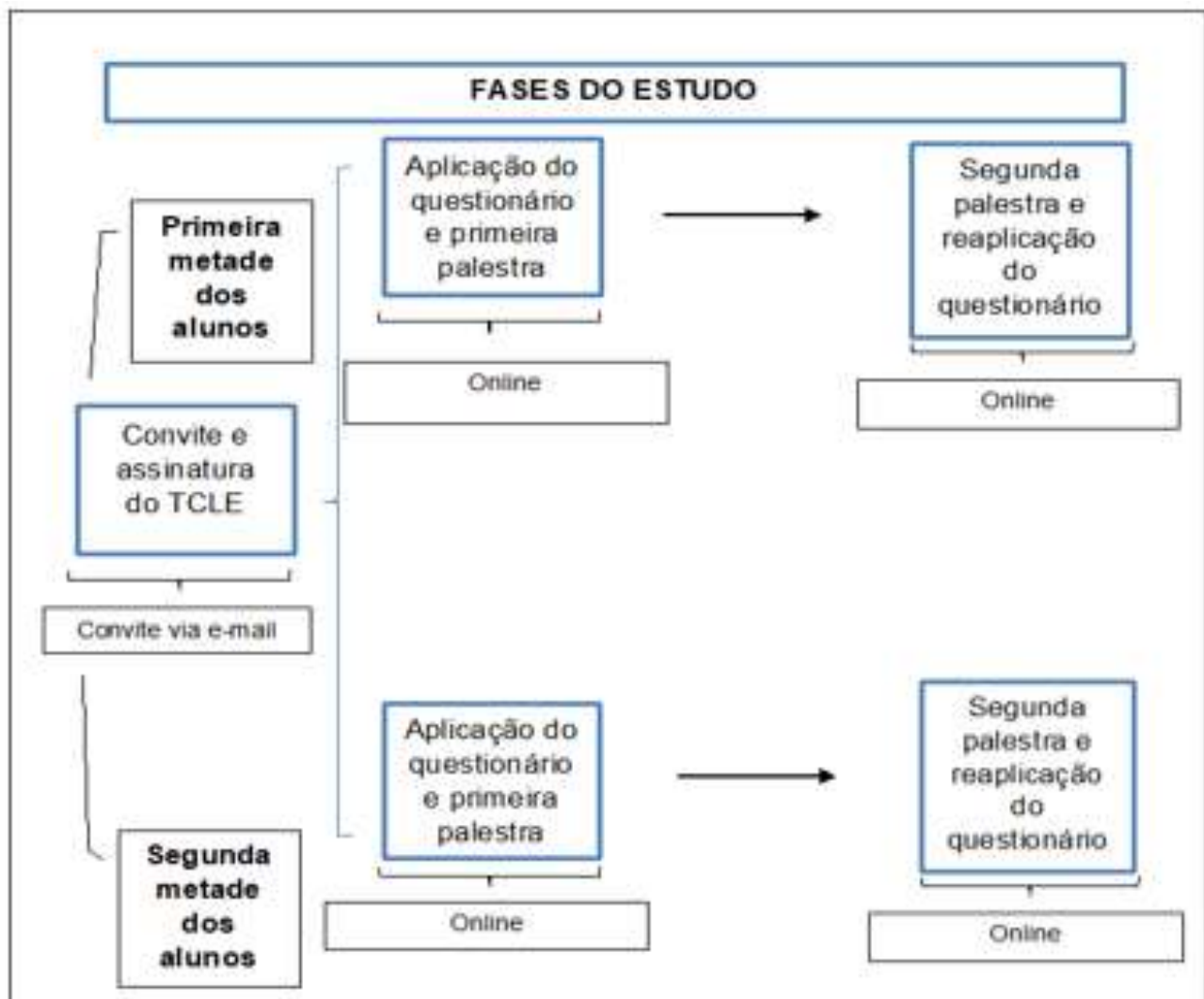

Fonte: Autores (2021).

\subsection{Questionário pré e pós ciclo de palestras}

O questionário aplicado aos alunos era composto por seis perguntas com quatro alternativas cada, sendo que apenas uma delas era a correta. Quatro perguntas eram conceituais, abordando as principais características da ECMO (definição, tipos, benefícios e complicações). Como forma de imergir os alunos na importância prática do tema, duas questões basearam-se em casos clínicos nos quais era necessário avaliar a possibilidade de uso de ECMO e se havia alguma contraindicação.

\subsection{Ciclo de palestras}

Após a primeira aplicação do questionário, foram ministradas duas palestras via plataforma Google Meet (online) de aproximadamente 10 minutos cada. A primeira palestra abordou generalidades sobre o ECMO, como sua definição, indicações, contraindicações, benefícios e complicações. Já a segunda palestra teve como foco o uso de ECMO em pacientes com COVID19, abordando tópicos como quais evidências sustentam seu uso e quais serviços brasileiros estão utilizando essa terapêutica atualmente.

\subsection{Análise estatística}

Os dados foram tabulados com o auxílio do Microsoft Excel versão 2018. Em seguida foi realizada uma análise descritiva para caracterizar o conhecimento dos alunos pré e pós a realização do ciclo de palestras oferecidos pelos autores desse estudo. Os dados foram apresentados em porcentagem, número absoluto, média e percentil.

A normalidade das variáveis contínuas foi testada pelo teste de Kolmogorov-Smirnov. Os dados foram considerados 
não paramétricos. Para comparação das variáveis categóricas entre os grupos foi realizado o teste de Wilcoxon. O nível de significância adotado foi de $5 \%$ e o software SPSS versão 22.0 foi usado para análise.

\section{Resultados}

Um total de oitenta alunos do primeiro ao sexto ano de medicina participaram desse estudo, entre eles, 61,3\% (N=49) eram do sexo feminino, 37,5\% ( $=30)$ do sexo masculino e 1,3\% (N=1) se identificou como outro. Destes, a média de idade foi de 23,24 anos $( \pm 3,37)$, variando de 20 a 44 anos. Quanto a distribuição de acordo com o ano da graduação observou-se que $67,5 \%(\mathrm{~N}=54)$ cursavam o quarto ano de medicina (Tabela 1$)$.

Tabela 1 - Caracterização da amostra.

\begin{tabular}{cr}
\hline Participantes $=80$ & \\
\hline Média de idade (anos) & $23,4( \pm 3,4)$ \\
\hline Gênero (\%) & 61,3 \\
\hline feminino & 37,5 \\
\hline masculino & 1,2 \\
\hline outro & 67,5 \\
\hline Ano de graduação (\%) & 20,0 \\
\hline quarto ano & 8,8 \\
\hline quinto ano & 1,3 \\
\hline terceiro ano & 2,6 \\
\hline primeiro ano
\end{tabular}

Fonte: Autores (2021).

Quanto ao número de acertos no questionário aplicado antes da realização do ciclo de palestras observou-se que a média de acertos foi de 3,89 variando de zero a seis pontos, com um percentil 50 de 4 pontos. Já após a realização do ciclo de palestras a média de acertos passou para 4,23 variando de três a seis pontos, com um percentil 50 de 4 pontos (Tabela 2).

Tabela 2 - Pontuação obtida no questionário pré e pós ciclo de palestras.

\begin{tabular}{|c|c|c|c|c|c|c|c|c|}
\hline & \multirow[b]{2}{*}{$\mathrm{N}$} & \multirow[b]{2}{*}{ Média } & \multirow[b]{2}{*}{$\begin{array}{c}\text { Estatística do } \\
\text { teste Padrão }\end{array}$} & \multirow[b]{2}{*}{ Mínimo } & \multirow[b]{2}{*}{ Máximo } & \multicolumn{2}{|c|}{ Percentis } & \multirow[b]{2}{*}{$75^{\circ}$} \\
\hline & & & & & & $25^{\circ}$ & $\begin{array}{c}50^{\circ} \\
\text { (Mediana) }\end{array}$ & \\
\hline $\begin{array}{l}\text { Nota pré } \\
\text { ciclo de } \\
\text { palestras }\end{array}$ & 53 & 3,89 & 1,251 & 0 & 6 & 3,00 & 4,00 & 5,00 \\
\hline $\begin{array}{l}\text { Nota pós } \\
\text { ciclo de } \\
\text { palestras }\end{array}$ & 53 & 4,23 & 0,750 & 3 & 6 & 4,00 & 4,00 & 5,00 \\
\hline
\end{tabular}

Fonte: Autores (2021).

Calculando-se o ponto médio da soma de pontos obtidas no questionário pré e pós ciclo de palestras observa-se que sem o ciclo de palestras o ponto médio de acertos foi de 10,83, já após a realização do ciclo de palestras passou para 14,91. Sendo que, o ponto médio é o ponto que divide o intervalo de classe em duas partes iguais. Ou seja, é a soma das classificações dividido pelo $\mathrm{N}$, como mostra a Tabela 3 . 
Tabela 3 - Ponto médio das notas obtidas no questionário pré e pós teste.

\begin{tabular}{|c|c|c|c|c|}
\hline & & $\mathrm{N}$ & Ponto médio & Soma de Classificações \\
\hline \multirow{4}{*}{$\begin{array}{l}\text { Nota pós ciclo de } \\
\text { palestras - Nota pré } \\
\text { ciclo de palestras }\end{array}$} & Classificações Negativas & $9^{a}$ & 10.83 & 97.50 \\
\hline & Classificações Positivas & $17^{\mathrm{b}}$ & 14.91 & 253.50 \\
\hline & Empates & $27^{\mathrm{c}}$ & & \\
\hline & Total & 53 & & \\
\hline
\end{tabular}

a. Nota pós ciclo de palestras < Nota pré ciclo de palestras

b. Nota pós ciclo de palestras > Nota pré ciclo de palestras

c. Nota pós ciclo de palestras $=$ Nota pré ciclo de palestras

Fonte: Autores (2021)

A fim de verificar a efetividade do ciclo de palestras para a aquisição de conhecimento dos acadêmicos de medicina sobre a ECMO foi realizado o teste de Wilcoxon que comprovou uma diferença estatística entre os pontos obtidos no questionário pré e pós a realização do curso ( $\mathrm{p}=0,039)$, como mostra a Tabela 4.

Tabela 4 - Teste de Wilcoxon

\begin{tabular}{lrr}
\hline & Nota pós ciclo de palestras - Nota pré ciclo de palestras \\
\hline$Z$ & $-2,067^{\mathrm{b}}$ \\
\hline Significância Sig. (2 extremidades) & 0,039 \\
\hline
\end{tabular}

\footnotetext{
a. Teste de Classificações Assinadas por Wilcoxon

b. Com base em postos negativos.

Fonte: Autores (2021).
}

\subsection{Palestras}

\section{Conteúdo programático do ciclo de palestras online - ECMO e COVID-19}

\section{Palestra geral de ECMO:}

- $\quad$ que é ECMO

- Panorama da utilização da ECMO no Brasil

- Quais os tipos

- Indicações

- Contra-indicações

- Complicações

\section{Palestra específica de ECMO para pacientes com COVID-19:}

- Utilização da ECMO nos pacientes com COVID-19

- Há evidências que sustentem o uso?

- Quando indicar?

- Quando contraindicar?

- Quais as possíveis complicações?

- Quais serviços brasileiros estão oferecendo essa tecnologia para pacientes com COVID-19?

Os assuntos abordados no presente estudo, na forma de palestras, foram de extrema importância para a formação dos estudantes participantes, já se referem a um tema inovador, recente e cuja abordagem não compõe a grade curricular atual desses alunos.

Durante a ministração das palestras, o interesse dos alunos em participar e tirar dúvidas foi nítido e, além disso, 
muitos relataram a importância do tema ao término da reunião virtual. Foi levantada a necessidade de abordagens semelhantes durante a graduação, principalmente sobre assuntos atuais que, muitas vezes não são abordados rotineiramente.

Para que mais alunos possam ter acesso ao conhecimento quanto ao uso de ECMO e pacientes com COVID-19, as palestras ministradas foram gravadas e ficarão disponíveis de forma permanente através dos links abaixo:

\author{
Palestra 1 \\ https://drive.google.com/file/d/1AUlkGpFzDnlVtdBXP_9Eqfx6pWKS2YEb/view?usp=sharing
}

\title{
Palestra 2
}

https://drive.google.com/file/d/1XB_BMYQBAvKOya0h7KaDh9GX0mv5aAq3/view?usp=sharing

\section{Discussão}

Em nossa pesquisa, 80 acadêmicos de medicina responderam ao questionário pré e pós-palestras com perguntas referentes ao uso de ECMO como suporte para o COVID-19 e assistiram as palestras propostas. Dentre todos os participantes, $90 \%$ já estavam na segunda metade do curso. Isso reflete que os acadêmicos tendem a participar de pesquisas do gênero quando já estão mais avançados no curso, momento em que possuem mais interesse e desejo de aperfeiçoamento sobre temas relevantes para a prática médica, como é o caso do uso de ECMO no COVID-19.

A ministração de palestras mostrou-se efetiva na transmissão de informações sobre o ECMO em pacientes com COVID, pois houve diferença significativa entre os pontos obtidos no questionário antes e após as duas palestras. Resultado semelhante foi encontrado por Fernandes e colaboradores (2016) que também ministraram palestras sobre um tema médico e compararam as respostas pré e pós-palestra, identificando uma maior porcentagem de acerto na maioria das questões aplicadas. Em contrapartida, a pandemia causada pelo coronavírus é recente e muitos estudos sobre o tema estão em andamento, principalmente os que avaliam possíveis abordagens terapêuticas. Dessa forma, o conhecimento acerca da doença ainda está em construção e as escolas médicas aos poucos introduzem o tema em sua grade curricular.

No contexto da ECMO como terapia de suporte em pacientes com COVID-19 e hipoxemia refratária, a terapia vem ganhando destaque nos últimos meses, porém seu uso no Brasil ainda é restrito a poucos centros (Latam, 2020). Além disso, o conhecimento a respeito de sua função, indicações e complicações ainda é faltoso, o que deixa claro a necessidade de promover discussões e disseminação de informações sobre o tema. Nesse caminho, muitas vezes, durante a graduação em medicina, certos temas não são abordados na grade curricular tradicional, o que pode deixar lacunas na construção do conhecimento dos alunos. Isso torna necessário a inserção da educação extracurricular, que complementa e favorece a formação médica.

Em uma pesquisa realizada na Universidade Federal de Santa Catarina, Pereira e colaboradores (2017) observaram que 59,26\% dos alunos reconhecem a importância das atividades extracurriculares para incrementar o conhecimento e as habilidades acadêmicas. Além disso, mais da metade dos estudantes possuíam uma percepção positiva referente a temas extracurriculares, tendo como reflexo um melhor desempenho nas provas, aumento do escore e maior acesso ao conhecimento. Resultado similar foi observado por Bussolotti e colaboradores (2016), no qual 64,3\% dos alunos afirmaram que a participação em atividades extracurriculares contribui para enriquecer o processo de ensino-aprendizagem. Dessa forma, percebe-se a importância da inserção de temas extracurriculares na formação médica, principalmente a respeito de assuntos atuais, como é o caso de novas abordagens terapêuticas para pacientes com COVID-19. 


\section{Conclusão}

O uso do ECMO no tratamento de pacientes com COVID-19 é uma terapia em ascensão e o conhecimento sobre sua aplicação por parte dos profissionais de saúde ainda está em progresso. O presente estudo demonstrou que a ministração de palestras foi uma metodologia efetiva em transmitir conhecimento a respeito dessa modalidade terapêutica para acadêmicos do curso de medicina. Recomenda-se que futuros estudos investiguem a aprendizagem dos alunos em outras áreas de conhecimento para confirmar a efetivação do conhecimento dos alunos em áreas afins.

\section{Referências}

Allen, S., Holena, D., Mccunn., Kohl., \& Sarani, B. (2011). A review of the fundamental principles and evidence base in the use of extracorporeal membrane oxygenation (ECMO) in critically ill adult patients. Journal of Intensive Care Medicine, 26(1), 13-26

Associação De Medicina Intensiva Brasileira (AMIB). (2020). Preparação de UTI para COVID-19: lições de experiências internacionais.

Barros, L., Rivetti, L. A., Furlanetto, B. H., Teixeira, E. M., \& Welikow. (2020) A. COVID-19: General guidelines for cardiovascular surgeons (standard guidelines - subject to change). Brazilian Journal of Cardiovascular Surgery, 35(2), 1-3

Beyls, C., Huette, P., Abou-Arab, O., Berna, P., \& Mahjoub, Y. (2020). Extracorporeal membrane oxygenation for COVID-19-associated severe acute respiratory distress syndrome and risk of thrombosis. BJA: British Journal of Anaesthesia, 125(2), 206-262.

Bussolotti, J. M.; Oliveira, M. R.; Pires, R. G.; \& Veiga, S. A. (2016). A importância das atividades complementares no processo de aprendizado: percepção dos alunos de cursos de educação a distância da universidade de Taubaté. Associação Brasileira de Ensino a Distância, 1-8.

Cunningham, A. C. ; Goh, H. P. ; \& Koh, D. (2020). Treatment of COVID-19: old tricks for new challenges. Critical Care, 24(1).

Estrela, C. (2018). Metodologia Científica: Ciência, Ensino, Pesquisa. Editora Artes Médicas.

Fernandes, M. A. M., Barroso, F. V. C., Damicio, Leite, W. S., \& Cerqueira, J. B. D. (2016). Análise do impacto de uma palestra sobre litíase urinária na Faculdade de Medicina (Liga de Urologia e Transplante Renal) Revista Encontros universitários da UFC, 1(1), 1-2.

Forrest, P., Ratchford, J., Burns, B., Herkers, R., Jackson, A., Plunkett, B., \& Pye, R. (2011). Retrieval of critically ill adults using extracorporeal membrane oxygenation: an Australian experience. Intensive Care Medicine, 37(5), 824-830.

Hill, J. D., O’Brien, T. G., Murray, J. J., Dontigny, L., Bramson, M. L., Osborn, J. J., \& Gerbode, F. (1972). Prolonged extracorporeal oxygenation for acute post-traumatic respiratory failure (shock-lung syndrome). Use of the Bramson membrane lung. The New England Journal of Medicine, 286 (12), 629-634.

Hong, X., Xiong, J., Feng, Z., \& Shi, Y., (2020). Extracorporeal membrane oxygenation (ECMO): does it have a role in the treatment of severe COVID-19? International Journal of Infectious Diseases, 94, 78-80

Huette, P., Byels, C., Guilbart, M., Coquet, A., Berna, P., Haye, G., \& Mahjoub, Y. (2020). Extracorporeal membrane oxygenation for respiratory failure in COVID-19 patients: outcome and time-course of clinical and biological parameters. Canadian Journal of Anaesthesia, 1-3.

Kon, Z. N., Smith,D. E., Chang, S., Goldenberg, R. M., Angel, L. F., Carillo, J. A., \& Galloway, A. C. (2021). Extracorporeal Membrane Oxygenation Support in Severe COVID-19. The Annals of Thoracic Surgery, 111(2), 537-543.

Latam, E. ELSO Guidance Document: ECMO for COVID-19. ELSO Latino-Americana (2020).

Marodin, G.; \& Goldim, J. R. (2009). Confusões e ambiguidades na classificação de eventos adversos em pesquisa clínica. Revista da Escola de Enfermagem da USP, 43, 690-696.

Patel, B. K. Insuficiência respiratória hipoxêmica aguda (IRHA, SARA). (2020). Manual MSD

Pereira, A. F. A., Conceição, D. S. R., Silva, M. C., Mota, B. C., \& Oliveira. P. R. S. (2017). A importância das atividades extracurriculares no desempenho acadêmico de estudantes da área de saúde. XI ENPEC, 1-11.

Ramantahan, K., Antognini, D., Combes, A., Paden, M., Zakhary, B., Ogino, M., \& Shekar, K. (2020). Planning and provision of ECMO services for severe ARDS during the COVID-19 pandemic and other outbreaks of emerging infectious diseases. The Lancet Respiratory Medicine, 8(5), 518-526.

Tramm, R., Ilic, D., Davies, A.R., Pellegrino, V.A., Romero, L., \& Hodgson, C. (2015). Extracorporeal membrane oxygenation for critically ill adults. The Cochrane Database of Systematic Reviews, 1, CD010381

World Health Organization. (2020). Clinical management of severe acute respiratory infection when novel coronavirus (2019-nCoV) infection is suspected: interim guidance.

Yang, X., Cai, S., Luo, Y., Zhu, F., Hu, M., Zhao., \& Peng, Z. (2020). Extracorporeal Membrane Oxygenation for Coronavirus Disease 2019-Induced Acute Respiratory Distress Syndrome: A Multicenter Descriptive Study. Critical Care Medicine, 48(9), 1289-1295.

Zeng, Y., Cai, Z., Zianyu, Y., Yang, B.X., Song, T., \& Yan, Q. (2020). Prognosis when using extracorporeal membrane oxygenation (ECMO) for critically ill COVID-19 patients in China: a retrospective case series. Critical Care, 24(1), 148 